\title{
To treat or not to treat: balancing therapeutic outcomes, toxicity and quality of life in patients with recurrent and/or metastatic head and neck cancer
}

\begin{abstract}
Barbara A. Murphy, MD
Vanderbilt Ingram Cancer Center, Nashville, Tennessee

There are a number of challenges facing head and neck cancer patients who present with metastatic or locally recurrent head and neck cancer; such as, limited treatment options, overall poor prognosis, and high symptom burden secondary to tumor and treatment. Disease and symptom management can be difficult, and requires that the potential benefits versus the adverse effects of systemic therapy be weighed very carefully. Individual patient characteristics including performance status, weight loss, symptom burden, comorbidities, and social supports must be taken into consideration. Unfortunately, reliable data describing the impact of therapy on symptom burden and quality of life (QOL) is lacking. Recently completed randomized phase III treatment trials have demonstrated the feasibility of incorporating patient reported outcome measures to assess symptoms and $Q O L$ into clinical studies. Nonetheless, obstacles to accurate and thorough QOL reporting remain. Development of tools directed at symptom burden and functional impairment in the metastatic or recurrent head and neck cancer population is needed. Such tools would enhance our ability to assess the impact of treatment, thus optimizing treatment decisions for patients with recurrent and/ or metastatic head and neck cancer.
\end{abstract}

$\mathrm{S}$ quamous cell carcinomas of the head and neck account for $3 \%$ of all new cancers diagnosed annually within the United States. ${ }^{1}$ According to the Surveillance Epidemiology and Ends Reports (SEER) database, 79\% of patients in the US present with local or regional advanced disease and are treated with combinedmodality therapy. ${ }^{2}$ Factors that influence treatment decision making include the following: resectability, function preservation, local patterns of care, and patient characteristics or preferences. In this cohort of patients, disease eradication is the goal of therapy. Conversely, for approximately $16 \%$ of patients who are diagnosed with metastatic disease at presentation, or the substantial portion of patients who develop non-curable disease recurrence, the main therapeutic objectives are palliation and prolongation of survival (accessible at

\footnotetext{
Manuscript received March 16, 2012; accepted August 13, 2013. Correspondence Barbara A. Murphy, MD, 2220 Pierce Avenue, 777 Preston Research Building, Nashville, TN 372326307 (barbara.murphy@Vanderbilt.Edu).

Disclosures Dr Murphy is a consultant for Bristol Myers Squib and Actogenix. Dr Murphy receives clinical trials support from Actogenix.

J Support Oncol 2013;11:149-159 @ 2013 Frontline Medical Communications
} DOI: 10.12788/i.suponc.0005 http://seer.cancer.gov/statfacts/html/oralcav. html). ${ }^{2,3}$ We define patients as having non-curable recurrence if development of metastatic disease or development of local recurrence is not amenable to either surgical resection or re-irradiation therapy.

Several changes in the epidemiology and treatment of metastatic and recurrent head and neck cancer (M/RHNC) have resulted in paradigm shifts that effect treatment decision making in this population. First, a combination of standard chemotherapy with cetuximab has demonstrated a survival advantage. This is the first time that any agent or combination of agents has demonstrated superiority in the treatment of M/RHNC. ${ }^{4}$ Second, human papilloma virus (HPV)-associated oropharyngeal cancers are epidemic in many areas of the world. The cohort of HPV-positive patients has an excellent prognosis with currently available primary treatment regimens. The recurrence rate in this population is low; however, data regarding the treatment responsiveness of $\mathrm{HPV}$-associated tumors that recur after primary therapy is lacking. Finally, with the increased use of aggressive combined modality regimens as primary therapy, patients with recurrent disease are often heavily pretreated and suffer from symptoms secondary to their initial therapy. It is important to understand 


\section{Review}

A

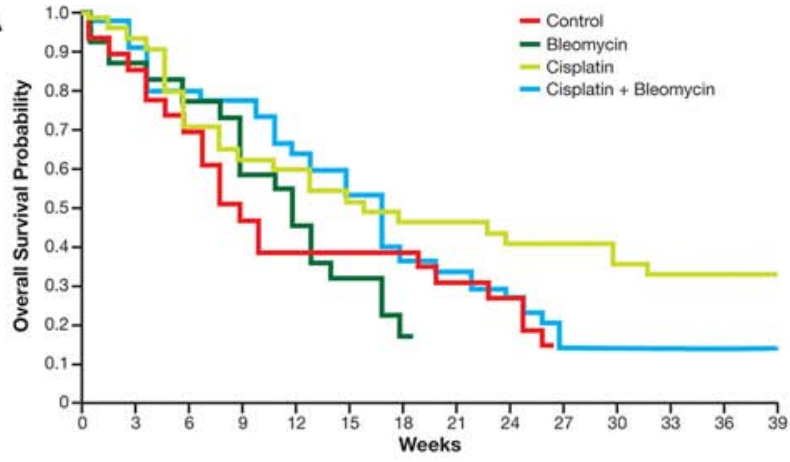

C

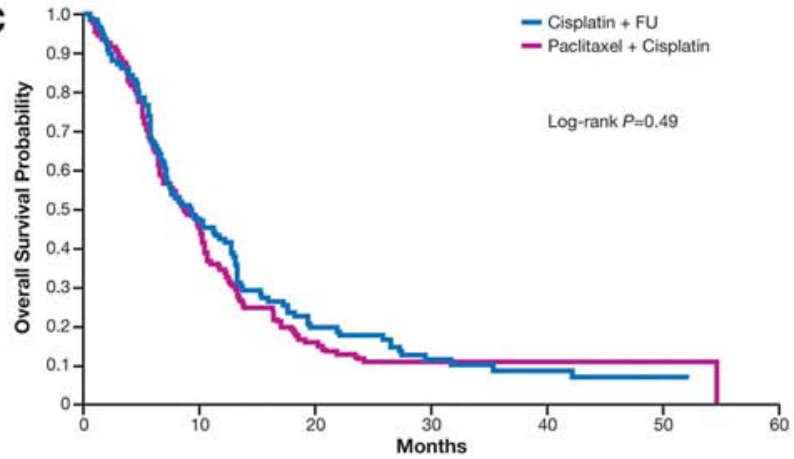

B

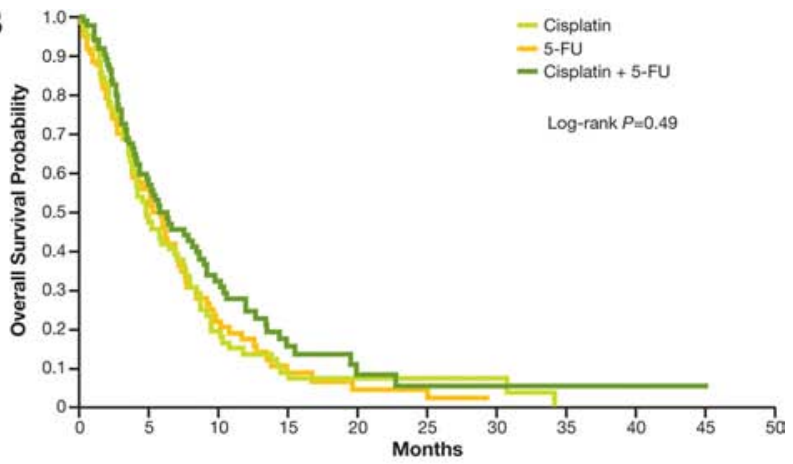

D

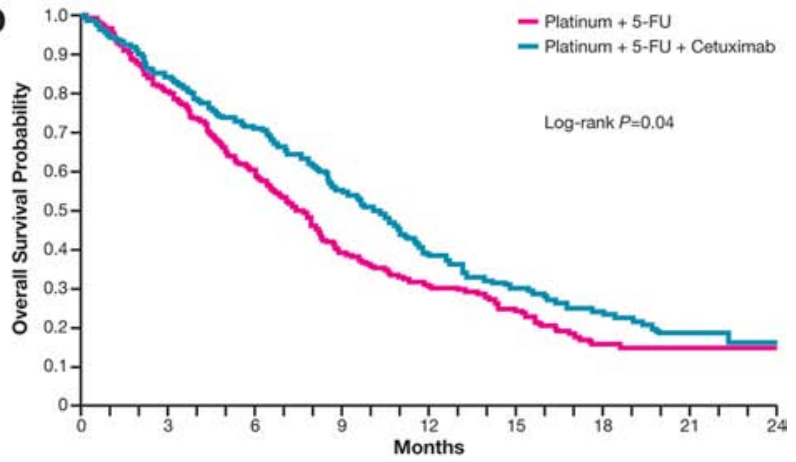

FIGURE Evolution of overall survival results from key trials in first-line R/M-HNC. A. Morton et al ${ }^{5}$ reported a significant advantage for single-agent cisplatin over BSC $(P<.05)$, but no advantage was noted for the addition of bleomycin to cisplatin versus cisplatin alone. B. Jacobs et al ${ }^{8}$ reported no significant survival advantages with the addition of $5 \mathrm{FU}$ to cisplatin. C. Gibson et al ${ }^{11}$ were unable to demonstrate a survival advantage for the substitution of 5-FU with paclitaxel in a cisplatin doublet. D. The EXTREME trial ${ }^{4}$ was the first randomized study to demonstrate a significant benefit for a combination regimen versus standard platinum therapy. Median OS was improved by 2.7 months (HR $=0.80 ; 95 \% \mathrm{Cl}$ : 0.64-0.99).

how the evolving epidemiology and treatment paradigms affect decision making for our patients. This requires an understanding of how these changes affect both the benefits and risks to the patient.

There are numerous manuscripts and reviews that address the efficacy of treatment regimens in $\mathrm{M} / \mathrm{RHNC}$ in terms of disease control. Clinical trials in this setting usually report toxicity using the Common Toxicity Criteria for Adverse Events. However, there are many shortcomings to toxicity assessment and reporting, including passive data collection; inaccurate grading of symptoms by data managers and nurses; and selective toxicity reporting, which is most evident by the failure to report clinically significant grade 1 and 2 toxicities. Furthermore, there is comparatively little data regarding the biopsychosocial impact of treatment. In order to make an informed decision, both patients and health care providers must understand the risks and benefits in terms of disease control and treatment toxicity, and the impact of treatment on general well-being, symptom burden, and functionality. This review will discuss the recent therapeutic progress in advanced-stage head and neck cancer (HNC) with an emphasis on the impact of treatment on symptom burden, functionality, and quality of life (QOL). Available QOL data from randomized controlled trials in the M/RHNC population and the challenges faced by supportive care researchers will be reviewed. Finally, we will discuss the considerations in making a treatment decision that balance optimal disease outcome with maximal QOL and minimal symptom burden.

\section{Current therapy for M/RHNC}

The prognosis and the survival benchmarks for patients with advanced-stage $\mathrm{HNC}$ are poor. Although heavily biased by patient selection, data would indicate that median survival for patients with untreated $\mathrm{M} / \mathrm{RHNC}$ is approximately 3 to 4 months. Only one randomized trial of chemotherapy versus best supportive care (BSC) has been conducted in this patient population. In this trial, BSC was compared with single-agent cisplatin and single-agent bleomycin (Figure A). ${ }^{5}$ The study was heavily flawed and insufficiently powered; nonetheless, a survival advantage for single-agent cisplatin over BSC was demonstrated. During the subsequent 2 decades, studies in the M/RHNC population centered on identification of new active chemotherapeutic agents and the development of combination regimens that were both tol- 
TABLE 1 Efficacy of first-line treatments in R/M-HNC

\begin{tabular}{|c|c|c|c|c|}
\hline Reference & Treatment regimen & $\mathbf{N}$ & $\mathbf{R R}(\%)$ & OS (mo) \\
\hline \multirow[t]{2}{*}{ Grose et al, $1985^{\circ}$} & Cisplatin & 50 & 8 & 4.5 \\
\hline & Methotrexate & 50 & 16 & 5.0 \\
\hline \multirow{2}{*}{ Forastiere et al, $1992^{7}$} & Carboplatin + 5-FU & 86 & $21^{a}$ & 5.0 \\
\hline & Methotrexate & 88 & 10 & 5.6 \\
\hline \multirow[t]{2}{*}{ Jacobs et al, $1992^{8}$} & Cisplatin +5 -FU & 79 & $32^{a}$ & 5.5 \\
\hline & 5-FU & 83 & 13 & 6.1 \\
\hline \multirow[t]{3}{*}{ Clavel et al, $1994^{9}$} & $\begin{array}{c}\text { Cisplatin + Methotrexate + } \\
\text { Bleomycin + Vincristine }\end{array}$ & 127 & $34^{a}$ & 6.5 \\
\hline & Cisplatin + 5-FU & 116 & $33^{a}$ & \\
\hline & Cisplatin & 122 & 16 & \\
\hline Gibson et al, $2005^{11}$ & Cisplatin + Paclitaxel & 100 & 26 & 8.1 \\
\hline \multirow[t]{2}{*}{ Vermorken et al, $2008^{4}$} & Platinum + 5-FU & 220 & 20 & 7.4 \\
\hline & Cetuximab + Platinum +5 -FU & 222 & $36^{a}$ & $10.1^{a}$ \\
\hline \multirow[t]{2}{*}{ Vermorken et al, $2013^{14}$} & Cisplatin + 5-FU & 330 & 25 & 9.0 \\
\hline & Panitumumab + Cisplatin $+5-\mathrm{FU}$ & 327 & $36^{a}$ & 11.1 \\
\hline \multirow[t]{2}{*}{ Urba et al, $2010^{12}$} & Cisplatin + Pemetrexed & 398 & 12 & 7.3 \\
\hline & Cisplatin + Placebo & 397 & 8 & 6.3 \\
\hline
\end{tabular}

Abbreviations: 5-FU, 5-fluorouracil; IFN- $\alpha 2$ b, interferon- $\alpha 2 b$; RR, response rate; OS, overall survival.

a Statistically significant difference.

erable and effective. Although interest in cytotoxic agents continues, the role of targeted agents has become an area of intense investigation.

\section{Cytotoxic therapy}

Historically, cytotoxic chemotherapy has been the firstline treatment of choice for patients with metastatic and/or recurrent disease for which local therapy is no longer suitable. Table 1 summarizes data from numerous clinical trials investigating various chemotherapeutic agents in the first-line setting. Unfortunately, chemotherapy is only modestly effective in this setting, with median survival rarely exceeding 9 months. Furthermore, few studies have reported response rates (RRs) greater than $40 \%$. Finally, randomized trials comparing monotherapy versus combination therapy have been unable to confirm the superiority of any one regimen versus another (Figures $\mathrm{B}$ and C) ${ }^{6-12}$ In fact, although RRs for cisplatin combined with 5-fluorouracil (5-FU) were consistently superior to other combinations and single agents in randomized trials, median survival durations did not significantly improve in a corresponding manner (Table 1). Emergence of taxanes did little to advance the therapeutic landscape: a randomized phase III study revealed no differences in either response or survival between the standard cisplatin/ 5-FU regimen and cisplatin/paclitaxel (Figure C). ${ }^{11}$ The toxicity profile was notably different with diarrhea, stomatitis, and hematological toxicities such as leukopenia and thrombocytopenia reported less frequently in patients treated with cisplatin/paclitaxel. Similarly, in a placebocontrolled trial, cisplatin and pemetrexed failed to demonstrate a statistically significant improvement in survival when compared to cisplatin in an intent-to-treat population; moreover, the RR and median overall survival (OS) were numerically lower than historical data with cisplatin/5-FU. ${ }^{12}$

Options for second-line treatment for patients with M/RHNC are very limited. Due to poor performance status and high symptom burden, many patients receive only BSC after failure of first-line therapy. ${ }^{13}$ For those who are candidates for further treatment, defined standards of care are lacking. Multiple single-arm and randomized trials have attempted to improve patient out- 
TABLE 2 Efficacy of R/M-HNC treatment regimens in the second-line or beyond

\begin{tabular}{|c|c|c|c|c|}
\hline Reference & Treatment regimen & $\mathbf{N}$ & RR (\%) & OS (mo) \\
\hline Cohen et al, $2003^{18}$ & Gefitinib & 47 & 10.6 & 8.1 \\
\hline Soulieres et al, $2004^{20}$ & Erlotinib & 115 & 4.3 & 6.0 \\
\hline Cohen et al, $2005^{19}$ & Gefitinib & 70 & 1.4 & 5.5 \\
\hline Herbst et al, $2005^{28}$ & Cetuximab + Cisplatin & 130 & 13 & $4.3-11.7^{a}$ \\
\hline Vermorken et al, $2007^{29}$ & Cetuximab & 103 & 13 & 5.7 \\
\hline \multirow[t]{3}{*}{ Stewart et al, $2009^{22}$} & Gefitinib $250 \mathrm{mg} /$ day & 158 & 2.7 & 5.6 \\
\hline & Gefitinib $500 \mathrm{mg} /$ day & 167 & 7.6 & 6.0 \\
\hline & Methotrexate & 161 & 3.9 & 6.7 \\
\hline \multirow[t]{2}{*}{ Argiris et al, $2009^{21}$} & Docetaxel + Placebo & 136 & 6 & 6.0 \\
\hline & Docetaxel + Gefitinib & 134 & 14 & 6.8 \\
\hline Fury et al, $2011^{23}$ & Cetuximab q2w $750 \mathrm{mg} / \mathrm{m}^{2}$ & 26 & 11 & \\
\hline \multirow[t]{2}{*}{ Machiels et al, $2011^{31}$} & BSC + Zalutumumab & 191 & 6.3 & 6.7 \\
\hline & BSC + Optional Methotrexate & 95 & 1.1 & 5.2 \\
\hline
\end{tabular}

Abbreviations: BSC, best supportive care; CT, chemotherapy; ITT, intent-to-treat; OS, overall survival; Pt, platinum; RR, response rate.

a By cohort; ITT OS not reported.

${ }^{b}$ Following objective response with single-agent cetuximab.

come in this setting with variable success; until recently, no randomized trial had demonstrated a statistically significant OS advantage for one regimen versus another beyond the first-line setting (Table 2). Outcomes with systemic therapy are generally poor: RRs are generally less than $10 \%$, responses are usually of short duration, and median survival is brief, with most patients succumbing to their cancer within 3 to 4 months.

\section{EGFR as a therapeutic target}

The epidermal growth factor receptor (EGFR) is a member of the transmembrane human epidermal growth factor receptor (HER) family of tyrosine kinase receptors whose multiple functions include regulation of cell growth, apoptosis, and differentiation. Dysregulation of the EGFR pathway, which primarily involves the phosphatidylinositol-3 kinase (PI3K) and the mitogenactivated protein kinase (MAPK) signaling networks, can result in substantial aberrations in normal cell function and can lead to tumor development by inhibiting apoptosis, promoting proliferation and angiogenesis, and prohibiting DNA repair mechanisms. EGFR is overexpressed in many solid malignancies, including HNC, where over $90 \%$ of tumors express levels of protein detectable by immunohistochemistry (IHC), typically at higher levels than normal tissues; high EGFR protein levels have been consistently associated with poor prognosis in locally advanced disease. ${ }^{15}$

\section{EGFR tyrosine kinase inhibitors}

Gefitinib and erlotinib are orally available inhibitors of EGFR tyrosine kinase activity (TKI), although the need for drug administration via feeding tube may act as a barrier for this type of treatment for some patients with $\mathrm{M} / \mathrm{RHNC}$. In phase I studies, EGFR TKIs presented a predictable and tolerable safety profile. ${ }^{16,17}$ However, subsequent phase II trials in refractory patients have demonstrated only limited activity for the single agents, with objective RRs of $1.4 \%$ to $10.6 \%$ for gefitinib, ${ }^{18,19}$ and $4.3 \%$ for erlotinib ${ }^{20}$ (Table 2). Unfortunately, randomized phase III trials with gefitinib have failed to demonstrate improved overall survival. A recent study in a mixed patient population of patients with M/RHNC (poor performance status and no prior treatment OR treatment refractory with good performance status) was terminated on interim analysis because it was deemed highly unlikely that the addition of gefitinib to docetaxel would improve OS compared to placebo. ${ }^{21}$ In a separate phase III trial in refractory patients, no significant differences in OS or RR were noted between patients treated with single-agent 
gefitinib (either 250 or $500 \mathrm{mg} /$ day) or methotrexate. ${ }^{22}$ Thus, while EGFR TKIs may have some activity in the recurrent and/or metastatic setting, their clinical role remains unclear.

\section{EGFR monoclonal antibodies}

The monoclonal antibodies (mAbs) cetuximab and panitumumab target the extracellular domain of EGFR, competing for binding with natural EGFR ligands such as transforming growth factor- $\alpha$ (TGF- $\alpha$ ), amphiregulin and epiregulin. These agents are typically administered intravenously; panitumumab is generally given biweekly while weekly dosing is standard for cetuximab. It should be noted that recent and ongoing studies investigating the merits of biweekly, high-dose cetuximab have not suggested compromised efficacy or safety compared to the standard regimen in HNC or colorectal cancer. ${ }^{23-26}$

A clinical role for cetuximab in $\mathrm{M} / \mathrm{RHNC}$ was initially described in phase II trials conducted in platinumrefractory disease. In this setting, cetuximab in combination with a platinum analogue yielded objective RRs of $10-13 \%{ }^{27,28}$ In another phase II trial, patients who had progressed on platinum-containing chemotherapy received cetuximab monotherapy; $13 \%$ of patients achieved a partial response, while $33 \%$ had stable disease. ${ }^{29}$ Those who experienced disease progression were eligible to receive salvage therapy with cetuximab in combination with a platinum agent. This group of patients, while achieving no objective responses, had a stable disease rate of $26 \%$. Together, these data suggested that cetuximab as a single agent could provide benefit in the second-line setting and beyond, for patients with otherwise limited therapeutic options.

On the basis of these promising results, the phase III EXTREME study was initiated to examine the potential efficacy and safety of cetuximab as first-line therapy, when added to a standard platinum-containing regimen. ${ }^{14}$ EXTREME demonstrated a significant improvement in median overall survival with the addition of cetuximab over platinum-based chemotherapy alone in the first-line treatment of M/RHNC (Figure D, Table 1). Specifically, the addition of cetuximab to 5-FU and either cisplatin or carboplatin produced a significant increase in best overall response to therapy (from $20 \%$ with platinum/ 5-FU alone to $36 \%$ with platinum $/ 5-\mathrm{FU}$ plus cetuximab; odds ratio 2.33 [95\% CI: $1.50-3.60] ; P<.001$ ) and a $36 \%$ improvement in median OS (from 7.4 to 10.1 months; Hazard Ratio $[\mathrm{HR}]=0.80$ [95\% CI: 0.64-0.99]; $P=$ .04). The risk of disease progression was also significantly reduced in the cetuximab arm: median progression-free survival (PFS) was prolonged by 2.3 months in patients receiving the cetuximab-containing regimen (from 3.3 to
5.6 months; $\mathrm{HR}=0.54$ [95\% CI: 0.43-0.67]; $P<.001$ ). Further analysis of this trial, using data collected from the European Organization for Research and Treatment of Cancer (EORTC) QLQ-30 and QLQ-H\&N35 assessment tools suggested that the addition of cetuximab did not change patients' QOL or social functioning. ${ }^{30}$

Another EGFR mAb, panitumumab, has been investigated in M/RHNC. Panitumumab is a fully human derivative of the immunoglobulin-G (IgG) 2 isotype, whereas cetuximab is a chimeric antibody derived from IgG1. While the relevance of these variations on their respective mechanisms of action has not been definitively elucidated, it has been postulated that fully human mAbs may limit antigenicity-related toxicities. On the other hand, it is thought that only IgG1 isotypes can mediate antibody-dependent cellular cytotoxicity (ADCC), which may have some effect on tumor shrinkage. The phase III SPECTRUM trial was designed to compare the effects of standard cisplatin/5-FU therapy with or without panitumumab in the first-line treatment of M/RHNC. The study failed to meet its primary endpoint of improved $\mathrm{OS},{ }^{14}$ although there were improvements in PFS and RR with panitumumab (Table 1). While it is intriguing to speculate that the activity of panitumumab fell short because of a lack of supportive anti-tumor ADCC, the human EGFR IgG1 mAb zalutumumab was also unable to improve OS versus $\mathrm{BSC} /$ methotrexate in a phase III trial in the second-line setting. ${ }^{31}$ Thus, cetuximab remains the only targeted agent to date to demonstrate an OS benefit in a randomized trial of M/RHNC.

\section{EGFR-targeting agents in development}

Two agents of note are being developed for clinical use in HNC: nimotuzumab, a humanized IgG1 mouse mAb, and afatinib (BIBW 2992), a small molecule inhibitor of EGFR and HER2. Although no clinical studies are currently ongoing with nimotuzumab in $M / R$ disease, preliminary data in the locally advanced setting have suggested that skin toxicity, the most common adverse event associated with anti-EGFR therapy, is limited with the use of this agent, possibly due to a lower binding affinity of the drug for the receptor. ${ }^{32}$

Afatinib, an orally administered, irreversible inhibitor of EGFR and HER2, was compared to cetuximab in a randomized phase II study in M/RHNC patients who had progressed on platinum-based therapy. ${ }^{33}$ Although adverse events such as diarrhea and dehydration occurred more frequently in those treated with afatinib, the RR was greater $(14.5 \%$ vs $3.2 \%)$ and compared favorably with other TKIs (Table 1). This promising agent is being tested further in a phase III trial, where an estimated 474 patients with platinum-refractory $\mathrm{M} / \mathrm{RHNC}$ will be ran- 
domized to receive either afatinib or methotrexate, with a primary endpoint of PFS (clinicaltrials.gov identifier NCT01345682).

\section{Treatment decisions in M/RHNC}

In the setting of recurrent or metastatic disease, patient management can be especially complex. Patients frequently present with suboptimal baseline performance status, substantial co-morbidities, and the sequelae and cumulative effects of heavy pretreatment. These patient characteristics may render them less responsive to therapy and/or prone to therapy-related side effects. Although the first and foremost interest of both clinician and patient is the ability of a therapeutic intervention to improve survival, the achievement of an optimal therapeutic outcome requires a balance between efficacy and side effects. As noted previously, systemic therapy for M/RHNC provides a modest improvement in survival, with a small but significant cohort of patients experiencing survival at 2 and 3 years post-treatment. Considering the functional status of patients and the best treatment regimens for individuals are critical components of overall therapeutic decisions. Two important factors that enter into such clinical decisions are the expected adverse events of therapy, their competing effect with disease control goals, and their potential impact on symptoms that are strictly disease-related; and the prognostic and predictive factors that clinicians can use to tailor interventions to the individual condition of a given patient.

\section{Balancing therapeutic goals: disease outcome and quality of life}

The toxicity of treatment regimens in patients with $\mathrm{M} / \mathrm{RHNC}$ has been well documented. Table 3 lists the most common adverse effects reported in selected clinical trials of $\mathrm{M} / \mathrm{RHNC}$. Unfortunately, our knowledge regarding the effects of therapy on overall symptom burden, which reflects both tumor- and treatment-related toxicities, is fairly limited. Tumor-related adverse effects may include symptoms from local tissue invasion (such as pain, secretions, or open wounds) or worsening functional impairment secondary to obstruction or infiltration of tissue (such as dysphagia or airway obstruction). Patients may also suffer from neuropsychobehavioral symptoms secondary to tumor-associated, systemic inflammatory and neuroendocrine processes. Neuropsychobehavioral symptoms may be categorized into 4 domains: cognitive function, mood disorders (such as anxiety and depression), somatic symptoms (gastrointestinal [GI] symptoms such as early satiety and nausea) and neurovegetative symptoms (fatigue and anorexia). The presence and severity of tumor-related symptoms may also predict disease out- comes: a small retrospective chart review has recently shown that escalating severity of pain (as documented by analgesic use) correlated with OS in patients receiving palliative care in a hospice setting. ${ }^{34}$ Furthermore, patients with weight loss or poor performance status tend to respond poorly to treatment.

To complicate matters, tumor-related symptoms may also be superimposed on persisting symptoms or functional deficits related to prior therapy. For example, many patients with recurrent disease will complain of xerostomia which, in most instances, can be clearly related to prior radiation therapy. While supportive interventions such as a tracheostomy tube or a percutaneous endoscopic gastrostomy (PEG) feeding tube alleviate severe adverse cancer complications, these interventions may result in symptom control problems in and of themselves. In addition, they may contribute to the burden of care, hinder communication, and create a state of social isolation. ${ }^{34}$

\section{Prognostic and predictive factors in $\mathrm{M} / \mathrm{RHNC}$}

Unlike many other solid tumors, we have failed to identify predictive biomarkers for available therapies. Without biomarkers indicating those $\mathrm{HNC}$ patients who have a greater likelihood of response, overall treatment benefit remains modest. A paradigm shift has occurred with the identification of the human papilloma virus (HPV) as an etiological factor in HNC. Patients with HPV-positive, locally advanced oropharyngeal cancer have improved survival and a smaller likelihood of developing recurrent disease. ${ }^{35,36}$ Despite this breakthrough, we lack the information to make treatment decisions tailored to this HPVpositive population in the locally advanced setting; and even less is known about the impact of HPV as a prognostic or predictive factor in recurrent and/or metastatic disease. Paradoxically, a recent analysis of the SPECTRUM trial suggested that adding the EGFR inhibitor panitumumab to chemotherapy could improve survival in patients with HPVnegative M/RHNC, while it had no impact on outcomes in HPV-positive patients. ${ }^{14}$ While interesting, these data are preliminary and do not warrant practice-altering interpretation. Nevertheless, close attention should be paid to new clinical trials that are now incorporating HPV status as a stratification factor in their designs. Forthcoming data from these trials should reveal any possible predictive value of this biomarker. The search for other predictive biomarkers is still ongoing but has, unfortunately, been thus far futile.

Other well-known prognostic factors are not embedded in our understanding of the etiology or molecular underpinnings of the disease, but rather in the characteristics of the patient and their treatment history. Many patients with $\mathrm{M} / \mathrm{RHNC}$ have a poor baseline perfor- 
TABLE $3 \mathrm{Gr} \geq 3 \mathrm{AE}$ in selected trials in $\mathrm{R} / \mathrm{M}-\mathrm{HNC}$

\begin{tabular}{|c|c|c|c|}
\hline Reference & $\mathbf{N}$ & Treatment regimen & Grade $\geq 3$ toxicity \\
\hline \multirow[t]{3}{*}{ Forastiere et al, $1992^{7}$} & \multirow[t]{3}{*}{270} & Cisplatin + 5-FU & $\begin{array}{l}\text { Hematologic, } 32.9 \% \\
\text { Diarrhea, } 2 \% \text {; Stomatitis, 14\%; Nausea/vomiting, } 8 \% \text {; } \\
\text { Peripheral Neuropathy, 1\%; Ototoxicity, } 4 \% \text {; Renal, } \\
9 \%\end{array}$ \\
\hline & & Carboplatin + 5-FU & $\begin{array}{l}\text { Hematologic, } 25.6 \% \\
\text { Diarrhea, } 2 \% \text {; Stomatitis, 15\%; Nausea/vomiting, 6\%; } \\
\quad \text { Renal, } 1 \%\end{array}$ \\
\hline & & Methotrexate & $\begin{array}{l}\text { Hematologic, } 16.1 \% \\
\text { Stomatitis, } 10 \% \text {; Nausea/Vomiting, 3\%; Renal, 3\% }\end{array}$ \\
\hline \multirow[t]{3}{*}{ Jacobs et al, $1992^{8}$} & \multirow[t]{3}{*}{249} & Cisplatin + 5-FU & $\begin{array}{l}\text { Leukopenia, 10\% } \\
\text { Vomiting, 35\%; Mucositis, 13\%; Ototoxicity, 3\% }\end{array}$ \\
\hline & & Cisplatin & $\begin{array}{l}\text { Leukopenia, } 1 \% \\
\text { Vomiting, } 18 \% \text {; Mucositis, } 2 \% \text {; Ototoxicity, } 1 \%\end{array}$ \\
\hline & & 5-FU & $\begin{array}{l}\text { Vomiting, } 5 \% \text {; Diarrhea, } 8.5 \% \text {; Mucositis, 13\%; } \\
\text { Ototoxicity, } 2 \%\end{array}$ \\
\hline \multirow[t]{2}{*}{ Gibson et al, $2005^{11}$} & \multirow[t]{2}{*}{218} & Cisplatin + 5-FU & $\begin{array}{l}\text { Hematologic, } 67 \% \\
\text { Infection, } 21 \% \text {; GU, 3\%; Nausea, 19\%; Vomiting, } \\
\text { 18\%; Diarrhea, 6\%; Stomatitis, 31\%; Hemorrhage, } \\
\text { 2\%; Mucositis, 1\%; Liver, 1\%; Cardiac, 2\%; } \\
\text { Hypotension, } 2 \% \text {; Neurosensory, } 2 \% \text {; Neuromotor, } \\
\text { 3\%; Metabolic, 15\%; Fatigue, 5\%; Dehydration, 5\% }\end{array}$ \\
\hline & & Cisplatin + Paclitaxel & $\begin{array}{l}\text { Hematologic, 55\% } \\
\text { Infection, 13\%; GU, 1\%; Nausea, 18\%; Vomiting, 10\%; } \\
\text { Diarrhea, 1\%; Liver, 3\%; Cardiac, 4\%; Hemorrhage, 1\%; } \\
\text { Hypotension, 5\%; Neurosensory, } 5 \% \text {; Neuromotor, 4\%; } \\
\text { Metabolic, 10\%; Fatigue, 7\%; Dehydration, 4\% }\end{array}$ \\
\hline \multirow[t]{2}{*}{ Vermorken et al, $2008^{4}$} & \multirow[t]{2}{*}{442} & Platinum-FU & $\begin{array}{l}\text { Neutropenia, 23\%; Anemia, 19\%; Thrombocytopenia, } \\
11 \% \text {; Leukopenia, } 9 \% \\
\text { Skin reactions, }<1 \% \text {; Hypokalemia, } 5 \% \text {; Cardiac } \\
\text { events, } 4 \% \text {; Vomiting, 3\%; Asthenia, 6\%; Anorexia, } \\
1 \% \text {; Hypomagnesemia, 1\%; Dyspnea, 8\%; } \\
\text { Pneumonia, } 2 \% \text {; Hypocalcemia, 1\%; Sepsis, }<1 \% \text {; } \\
\text { Tumor hemorrhage, 3\%; Respiratory failure, } 2 \%\end{array}$ \\
\hline & & Cetuximab + Platinum-FU & $\begin{array}{l}\text { Neutropenia, 22\%; Anemia, 13\%; Thrombocytopenia, } \\
11 \% \text {; Leukopenia, } 9 \% \\
\text { Skin reactions, } 9 \% \text {; Hypokalemia, 7\%; Cardiac events, } \\
\text { 7\%; Vomiting, 5\%; Asthenia, 5\%; Anorexia, 5\%; } \\
\text { Hypomagnesemia, 5\%; Dyspnea, 4\%; Pneumonia, } \\
\text { 4\%; Hypocalcemia, 4\%; Sepsis, } 4 \% \text {; Tumor } \\
\text { hemorrhage, 1\%; Respiratory failure, } 1 \%\end{array}$ \\
\hline \multirow[t]{2}{*}{ Argiris et al, $2009^{21}$} & \multirow[t]{2}{*}{270} & Docetaxel + Placebo & $\begin{array}{l}\text { Neutropenia, 3\%; Febrile neutropenia, } 1 \% \\
\text { Fatigue, } 15 \% \text {; Rash/desquamation, } 1 \% \text {; Anorexia, 2\%; } \\
\text { Dehydration, } 5 \% \text { : Diarrhea, } 2 \% ; \text { Nausea, } 4 \% \text {; } \\
\text { Vomiting, 3\%; Bleeding, 1\% }\end{array}$ \\
\hline & & Docetaxel + Gefitinib & $\begin{array}{l}\text { Neutropenia, 3\%; Thrombocytopenia, } 1 \% \\
\text { Fatigue, } 11 \% \text {; Anorexia, } 4 \% \text {; Dehydration, 5\%: Diarrhea, } \\
12 \% \text {; Nausea, } 5 \% \text {; Vomiting, } 2 \% \text {; Neuropathy-motor, } 1 \% \text {; } \\
\text { Neuropathy-sensor, 2\%; Bleeding, } 1 \%\end{array}$ \\
\hline \multirow[t]{2}{*}{ Urba et al, $2012^{12}$} & \multirow[t]{2}{*}{777} & Cisplatin + Pemetrexed & $\begin{array}{l}\text { Neutropenia, } 3 \% \text {; Febrile neutropenia, } 3 \% \\
\text { Fatigue, } 5 \% \text {; Anorexia, } 2 \% \text {; Nausea, } 3 \% \text {; Vomiting, } 2 \%\end{array}$ \\
\hline & & Cisplatin + Placebo & Fatigue, $2 \%$; Anorexia, 2\%; Nausea, 3\%; Vomiting, 3\% \\
\hline
\end{tabular}

mance status which, not surprisingly, appears to correlate with decreased survival (Table 4). In addition, the degree of decline in performance status during the course of treatment may be associated with a lack of response. ${ }^{27}$
The bulk of HNC patients with metastatic or recurrent disease have already been treated with primary combined modality therapy, which increasingly means that patients have received aggressive induction chemotherapy with 
TABLE 4 Baseline performance status and survival outcome in R/M-HNC

\begin{tabular}{|c|c|c|c|c|}
\hline Reference & PS stratification & $\mathbf{N}$ & \multicolumn{2}{|c|}{ OS measure } \\
\hline \multirow[t]{2}{*}{ Baselga et al, $2005^{27}$} & KPS $<80 \%$ & 30 & Median (days) & 131.5 \\
\hline & $K P S \geq 80 \%$ & 63 & & 216 \\
\hline \multirow{4}{*}{ Herbst et al, $2005^{28}$} & KPS $<80 \%$ & 6 & & 17 \\
\hline & $K P S \geq 80 \%$ & 45 & & 44 \\
\hline & Pts with PD & & & \\
\hline & $K P S \geq 80 \%$ & 51 & & 18 \\
\hline \multirow[t]{2}{*}{ Vermorken et al, $2007^{29}$} & KPS $<80 \%$ & 38 & Median (days) & 138 \\
\hline & $K P S \geq 80 \%$ & 64 & & 202 \\
\hline \multirow[t]{2}{*}{ Vermorken et al, $2008^{4}$} & KPS $<80 \%$ & 52 & $\mathrm{HR}$ & 1.14 \\
\hline & $K P S \geq 80 \%$ & 390 & & 0.75 \\
\hline
\end{tabular}

Abbreviations: ECOG, Eastern Cooperative Oncology Group; HR, hazard ratio; KPS, Karnofsky performance status; OS, overall survival; PD, progressive disease; PS, performance status; Pts, patients; SD, stable disease.

regimens such as docetaxel, cisplatin and 5-FU (TPF). These patients may have residual toxicities secondary to systemic chemotherapy, including peripheral neuropathy, renal insufficiency, and bone marrow suppression. Late toxicities from such regimens may limit the ability to administer treatment in the metastatic or recurrent setting, or may predispose patients to early development of toxicities with subsequent treatment. Weight loss, a common complication of HNC and its treatment, may have a negative impact on performance status and the ability to tolerate therapy. ${ }^{37}$

Many patients present with or develop comorbidities, including secondary malignancies. ${ }^{37}$ Comorbid disease has been established as a significant prognostic indicator of survival in HNC patients at all stages of disease, without regard for age, race, gender, or socioeconomic status. ${ }^{38,39}$ A retrospective study restricted to HNC patients with metastatic disease revealed a significant correlation between the severity of baseline comorbidities and increased mortality rates. ${ }^{40}$

Treatment history may also predict outcome. Patients who do not achieve a complete response to primary radiation regimens or who recur rapidly after completing therapy (within 6 months) have a lower RR to treatment for their recurrent disease, and clinical trials for $\mathrm{M} / \mathrm{RHNC}$ often exclude this subset of patients because of the refractory nature of their tumor. In addition, patients who develop recurrent disease in an area that had been previously irradiated have a worse prognosis. ${ }^{41}$
Other prognostic variables, including socioeconomic and sociodemographic circumstances, likely play a role in the incidence of and mortality from head and neck cancer. Lack of health care coverage and the higher prevalence of smoking in patients with low socioeconomic status may contribute to the poor prognosis of this subpopulation. ${ }^{42}$ Marital status may also correlate with outcome; several studies have suggested that unmarried or unpartnered patients are at a startling disadvantage for survival. ${ }^{42-44}$ In addition, psychosocial factors such as depression, anxiety, and stress are highly prevalent in HNC; depression, in particular, has been independently linked to reduced QOL, disease recurrence and poor overall survival. ${ }^{45-49}$ Whether psychosocial factors are causal for declining health, or are rather symptoms of it, is difficult to establish.

\section{Tools and measures of QOL and symptom burden issues for consideration}

Assessment of the effects of therapy on QOL and symptom control in M/RHNC patients undergoing treatment in the context of a clinical trial is challenging. The obstacles for establishing and validating robust tools are discussed below.

There are no measures that have been developed and validated to measure symptom burden in patients with M/RHNC. Currently available tools, such as the EORTC H\&N35 and Functional Assessment of Cancer Therapy (FACT)-HN questionnaires, include symptoms that are largely directed at patients undergoing primary com- 
bined modality therapy for locally advanced disease. Symptoms such as open wounds or tumor odor, which may be highly problematic in the metastatic setting, are missing.

Survival for many patients is short. Thus, there is a rapid attrition in numbers of patients completing QOL questionnaires as a part of treatment trials. Therefore, missing data becomes a major challenge to any statistical analysis, studies are hampered by lack of power, and meaningful comparisons between interventions tend to be weak.

Patients with $\mathrm{M} / \mathrm{RHNC}$ have high symptom levels and functional deficits. Some of these symptoms are secondary to progressive disease, while others are related to the late effects of prior therapy. While it is reasonable to expect that a tumor-related symptom may improve over time if there is a clinical response to therapy, one would not expect side effects secondary to radiation therapy, such as xerostomia or trismus, to improve with palliative chemotherapy. It is critical to understand which symptoms systemic therapy may and may not palliate. Unfortunately there is very limited literature on the type of symptom control issues experienced by patients with M/RHNC. In the future, we must develop a better understanding of the symptom burden in this cohort of patients and provide investigators with measurement tools that center on those symptoms that may be reversed with chemotherapy. With this type of information, we can provide clinicians and patients a more accurate picture of the palliative benefit of therapy.

Distinguishing the effects of therapy from the effects of cancer progression can be difficult. Progressive disease is likely to result in an increased symptom burden, which may cause a decrease in QOL. It is hoped that systemic therapy will result in tumor shrinkage with an associated relief of tumor-related symptom burden; however, treatment may itself be associated with substantial toxicity. Although attribution may be evident in some circumstances, the clinician is often faced with clinical problems of unclear etiology. For example, it may be difficult to determine whether worsening fatigue is due to treatment or tumor progression. One way of addressing this concern is to select appropriate time points for assessment. It is likely that the most informative data to separate the effects of treatment and progressive cancer will derive from the early phase of therapy, when tumor effects are minimized and the immediate, obvious effects of treatment may be discerned.

\section{Quality of life data in the recurrent/metastatic setting}

There are a limited number of large, randomized studies that have reported QOL, symptom, or functional outcomes in patients with $M / R$ disease. The first major study to report the results of a formal QOL analysis in this setting was E1395, a randomized comparison of cisplatin plus 5-FU $\left(\right.$ Arm A) versus cisplatin and paclitaxel $(\operatorname{Arm~B}) .{ }^{11,50} \mathrm{Al}-$ though the study failed to demonstrate a substantial difference in survival between treatment arms, some useful information could be gleaned from an analysis of $3 \mathrm{QOL}$ questionnaires: the FACT-General (FACT-G), the Head and Neck Subscale (HNS), and the Brief Pain Inventory (BPI). Patients completed the questionnaires at baseline and at 7 and 16 weeks after the start of therapy. Patients randomized to Arm A had a decrease in QOL over time in comparison to patients in $\operatorname{Arm~B~}(P=.04)$. No differences in the social, emotional or physical domains were demonstrated; however, patients in Arm B had improvement in the functional domain $(P=.036)$, HNS $(P=.014)$ and Toxicity Outcome Index (TOI; $P=.011)$. Hematological and GI toxicities were worse for patients treated with cisplatin/ 5-FU, although no clear correlation between toxicities and QOL was noted. Patients in Arm B experienced a significant improvement in worst pain compared to $\operatorname{Arm} \mathrm{A}(P=$ $.038)$, but average pain or interference was not markedly different between the treatment arms. Improvements in QOL and pain were transient. ${ }^{50}$

Subsequently, a phase III trial was conducted with single-agent gefitinib ( 250 or $500 \mathrm{mg} /$ day) or methotrexate $^{20}$ in refractory M/RHNC patients. No significant differences in OS or ORR were noted between the 3 arms. Comparison of QOL scores obtained using the FACT-H\&N questionnaire also revealed no significant variance between treatments: the mean change from baseline total scores were $13.4 \%, 18.0 \%$, and $6.0 \%$ for gefitinib $250 \mathrm{mg} /$ day, $500 \mathrm{mg} /$ day, and methotrexate, respectively. Symptom improvement scores obtained from the FACTH\&N Symptom Index (FHNSI-10) questionnaire, a symptom-focused index developed specifically for use in the $\mathrm{M} / \mathrm{RHNC}$ population, likewise demonstrated no significant benefit for any treatment arm, although the scores were numerically higher for the gefitinib arms. ${ }^{20}$ Thus, the marginal activity of EGFR TKIs does not translate into well-documented symptom relief outcomes.

In the landmark EXTREME trial, where patients were randomized to receive first-line chemotherapy (either cisplatin or carboplatin plus 5-FU) with or without cetuximab, QOL was specified as a secondary endpoint and was examined in detail in a subsequent publication. ${ }^{30}$ Data was collected from 2 questionnaires: the QOL Questionnaire-Core 30 (QLQ-30), which assesses overall quality of life, and the QLQ-H\&N35, which is comprised of head and neckspecific symptom scales. Patients were asked to complete the questionnaires at screening, at cycle 3 , at 6 weeks following therapy completion, at 6 and 12 months after randomization, and at the final tumor assessment; however, compliance 
levels were sufficient for statistical evaluation only at cycle 3 and at 6 months. On the QLQ-H\&N35 questionnaire at cycle 3, patients in the cetuximab arm reported improvements in pain $(P=.0083)$, swallowing $(P=.0034)$, speech $(P=.0029)$, and eating in public (social eating; $P=.0182)$. These values remained significant after adjusting for baseline values. Subscale analysis of the QLQ-30 at cycle 3 revealed that physical functioning, fatigue, nausea and vomiting, and pain were all significantly improved in the cetuximab arm; however, none of these parameters retained significance following baseline adjustment. At the 6-month assessment, the global health score calculated from the QLQ-30 was significantly higher in the cetuximab-containing arm compared to the chemotherapy only arm $(P=.0399)$. Although the difference did not remain significant after adjustment for baseline, pattern-mixture analysis suggested a significant effect for the addition of cetuximab to chemotherapy in improving the overall $\mathrm{QOL}(P=.0415)$. In total, the $\mathrm{QOL}$ analysis suggested that the addition of cetuximab to chemotherapy in the first-line setting did not negatively impact patients' QOL or social functioning.

Finally, a recent phase III, randomized trial compared cisplatin and pemetrexed to cisplatin plus placebo as a first-line treatment for M/RHNC patients. ${ }^{12}$ The study failed to meet its primary endpoint of OS, and analysis of QOL as a secondary endpoint revealed no significant changes from baseline, or in any individual symptoms, as assessed by FACT-H\&N.

\section{Conclusions}

Treatment decisions for patients with head and neck cancer with recurrent and/or metastatic disease require striking a balance between the potential survival benefits of a given therapeutic regimen and the possible detriment that such treatment may inflict on quality of life, both of which are dependent on the individual circumstances of the patient. The constraints of extensive comorbidities, long-term effects of heavy pre-treatment and other negative psychosocial and sociodemographic prognostic factors in the M/RHNC $\mathrm{pa}^{-}$ tient population places even greater limitations on the available therapeutic options in this setting. Cetuximab, concurrent with platinum-based chemotherapy, is the only regimen to have demonstrated efficacy over chemotherapy alone in a randomized trial - importantly, without a corresponding decrease in QOL. ${ }^{30}$ In the future, it is important to expand efforts to assess the impact of treatment on QOL and symptom burden in this frail, high-risk patient population. Unfortunately, validated tools directed at $\mathrm{M} / \mathrm{RHNC}$ have not been developed. Thus, development and testing of questionnaires with appropriate content are needed. Appropriately designed tools will provide clinicians with the data needed to maintain equilibrium between treatment efficacy and symptom control.

\section{Acknowledgments}

Editorial assistance for the preparation of this manuscript was provided by Clinical Insights Inc., supported by Bristol-Myers Squibb.

\section{References}

1. Jemal A, Siegel R, Xu J, Ward E. Cancer Statistics, 2010. CA Cancer J Clin. 2010;60(5):277-300.

2. National Cancer Institute. SEER Stat Fact Sheets: Oral Cavity and Pharynx. Surveillance Epidemiology and End Results. http://seer.cancer. gov/statfacts/html/oralcav.html. Accessed July 24, 2012.

3. Pfister DG, Ang KK, Brizel D, et al. National Comprehensive Cancer Network. NCCN Clinical Practice Guidelines in Oncology ${ }^{\mathrm{TM}}$ : Head and Neck Cancers 2012; version 1.2012. http://www.nccn.org/ professionals/physician_gls/pdf/head-and-neck.pdf. Published: April 26, 2012. Accessed July 24, 2012.

4. Vermorken JB, Mesia R, Rivera F, et al. Platinum-based chemotherapy plus cetuximab in head and neck cancer. $N$ Engl J Med. 2008;359:1116-1127.

5. Morton RP, Rugman F, Dorman EB, et al. Cisplatin and bleomycin for advanced or recurrent squamous cell carcinoma of the head and neck: a randomized factorial phase III controlled trial. Cancer Chemother Pharmacol. 1985;15(3):283-289.

6. Grose WE, Lehane DE, Dixon DO, Fletcher WS, Stuckey WJ. Comparison of methotrexate and cisplatin for patients with advanced squamous cell carcinoma of the head and neck region: a Southwest Oncology Group study. Cancer Treat Rep. 1985;69(6):577-581.

7. Forastiere AA, Metch B, Schuller DE, et al. Randomized comparison of cisplatin plus fluorouracil and carboplatin plus fluorouracil versus methotrexate in advanced squamous-cell carcinoma of the head and neck: a Southwest Oncology Group study. J Clin Oncol. 1992;10(8):1245-1251.

8. Jacobs C, Lyman G, Velez-García E, et al. A phase III randomized study comparing cisplatin and fluorouracil as single agents and in combination for advanced squamous cell carcinoma of the head and neck. J Clin Oncol. 1992;10(2):257-263.

9. Clavel M, Vermorken JB, Cognetti F, et al. Randomized comparison of cisplatin, methotrexate, bleomycin and vincristine (CABO) versus cisplatin and 5-fluorouracil $(\mathrm{CF})$ versus cisplatin $(\mathrm{C})$ in recurrent or metastatic squamous cell carcinoma of the head and neck. A phase III study of the EORTC Head and Neck Cancer Cooperative Group. Ann Oncol. 1994;5(6):521-526.

10. Schrijvers D, Johnson J, Jiminez U, et al. Phase III trial of modulation of cisplatin/fluorouracil chemotherapy by interferon alfa- $2 \mathrm{~b}$ in patients with recurrent or metastatic head and neck cancer. Head and Neck Interferon Cooperative Study Group J Clin Oncol. 1998;16(3):1054-1059.

11. Gibson MK, Li Y, Murphy B, et al. Randomized phase III evaluation of cisplatin plus fluorouracil versus cisplatin plus paclitaxel in advanced head and neck cancer (E1395): an intergroup trial of the Eastern Cooperative Oncology Group. J Clin Oncol. 2005;23(15):3562-3567.

12. Urba S, van Herpen CM, Sahoo TP, et al. Pemetrexed in combination with cisplatin versus cisplatin monotherapy in patients with recurrent or metastatic head and neck cancer: Final results of a randomized, double-blind, placebo-controlled, phase 3 study. Cancer. 2012;10(19):4694-4705

13. León X, Hitt R, Constenlaz M, et al. A retrospective analysis of the outcome of patients with recurrent and/or metastatic squamous cell carcinoma of the head and neck refractory to a platinum-based chemotherapy. Clin Oncol (R Coll Radiol). 2005;17(6):418-424.

14. Vermorken JB, Stohlmacher-Willimas J, Davidenko I, et al. Cisplatin and fluoruracil with or without panitumumab in patients with recurrent or metastatic squamous-cell carcinoma of the head and neck (SPECTRUM): an open-label phase 3 randomized trial. Lancet Oncol. 2013;14(8):697-710.

15. Grandis JR, Melhem MF, Gooding WE, et al. Levels of TGF-a and EGFR protein in head and neck squamous cell carcinoma and patient survival. J Natl Cancer Inst. 1998;90(11):824-832. 
16. Ang KK, Berkey BA, Tu X, et al. Impact of epidermal growth factor receptor expression on survival and pattern of relapse in patients with advanced head and neck carcinoma. Cancer Res. 2002; 62(24):7350-7356.

17. Ciardiello F, Tortora G. A novel approach in the treatment of cancer: targeting the epidermal growth factor receptor. Clin Cancer Res. 2001;7(10):2958-2970.

18. Cohen EE, Rosen F, Stadler WM, et al. Phase II trial of ZD1839 in recurrent or metastatic squamous cell carcinoma of the head and neck. J Clin Oncol. 2003;21(10):1980-1987.

19. Cohen EE, Kane MA, List MA, et al. Phase II trial of gefitinib $250 \mathrm{mg}$ daily in patients with recurrent and/or metastatic squamous cell carcinoma of the head and neck. Clin Cancer Res. 2005;11(23):8418-8424.

20. Soulieres D, Senzer NN, Vokes EE, Hidalgo M, Agarwala SS, Siu LL. Multicenter phase II study of erlotinib, an oral epidermal growth factor receptor tyrosine kinase inhibitor, in patients with recurrent or metastatic squamous cell cancer of the head and neck. J Clin Oncol. 2004;22(1):77-78.

21. Argiris A, Ghebremichael M, Gilbert J, Burtness B, Forastiere A. A phase III randomized, placebo-controlled trial of docetaxel (D) with or without gefitinib $(G)$ in recurrent or metastatic $(R / M)$ squamous cell carcinoma of the head and neck $(\mathrm{SCCHN})$ : A trial of the Eastern Cooperative Oncology Group (ECOG). J Clin Oncol. 2009;27(suppl 15):6011. 2009 ASCO Annual Meeting Proceedings (Post-Meeting Edition).

22. Stewart JS, Cohen EE, Licitra L, et al. Phase III study of gefitinib compared with intravenous methotrexate for recurrent squamous cell carcinoma of the head and neck [correction appears in J Clin Oncol. 2009;27(20):3410]. J Clin Oncol 2009;27(11):1864-1871.

23. Fury MG, Sherman EJ, Lisa DM, et al. A randomized phase II study of cetuximab (C) every 2 weeks at either 500 or $750 \mathrm{mg} / \mathrm{m}^{2}$ for patients $(\mathrm{Pts})$ with recurrent or metastatic $(\mathrm{R} / \mathrm{M})$ head and neck squamous cell cancer (HNSCC). J Clin Oncol. 2011;29 (suppl May 20, 2011):5563. ASCO Meeting Abstracts, Part 1; abstract 5563.

24. Ciuleanu T, Nikolic V, Shmueli E, et al. Cetuximab weekly (q1w) versus every two weeks (q2w) plus FOLFOX4 as first-line therapy in patients (pts) with KRAS wild-type (wt) metastatic colorectal cancer (mCRC). J Clin Oncol. 2011;29 (suppl May 20, 2011):5563. ASCO Meeting Abstracts, Part 1; abstract 3580.

25. Roca JM, Alonso V, Paricay C, et al. Cetuximab given every 2 weeks plus irinotecan is an active and safe option for previously treated patients with metastatic colorectal cancer. Chemotherapy. 2010;56(2):142-146.

26. Tabernero J, Ciardiello F, Rivera F, et al. Cetuximab administered once every second week to patients with metastatic colorectal cancer: a two-part pharmacokinetic/pharmacodynamic phase I doseescalation study. Ann Oncol. 2010;21(7):1537-1545.

27. Baselga J, Trigo JM, Bourhis J, et al. Phase II multicenter study of the antiepidermal growth factor receptor monoclonal antibody cetuximab in combination with platinum-based chemotherapy in patients with platinum-refractory metastatic and/or recurrent squamous cell carcinoma of the head and neck. J Clin Oncol. 2005;23:5568-5577.

28. Herbst RS, Arquette M, Shin DM, et al. Phase II multicenter study of the epidermal growth factor receptor antibody cetuximab and cisplatin for recurrent and refractory squamous cell carcinoma of the head and neck. J Clin Oncol. 2005;23(24):5578-5587.

29. Vermorken JB, Trigo J, Hitt R, et al. Open-label, uncontrolled, multicenter phase II study to evaluate the efficacy and toxicity of cetuximab as a single agent in patients with recurrent and/or metastatic squamous cell carcinoma of the head and neck who failed to respond to platinum-based therapy. J Clin Oncol. 2007;25(16):2171-2177.

30. Mesía R, Rivera F, Kawecki A, et al. Quality of life of patients receiving platinum-based chemotherapy plus cetuximab first line for recurrent and/or metastatic squamous cell carcinoma of the head and neck. Ann Oncol. 2010;21(10):1967-1973.

31. Machiels J-P, Subramanian S, Ruzsa A, et al. Zalutumumab plus best supportive care versus best supportive care alone in patients with recurrent or metastatic squamous-cell carcinoma of the head and neck after failure of platinum-based chemotherapy: an openlabel, randomised phase 3 trial. Lancet Oncol. 2011;12(4):333-343.

32. Ramakrishnan MS, Eswaraiah A, Crombet T, et al. Nimotuzumab, a promising therapeutic monoclonal for treatment of tumors of epithelial origin. MAbs. 2009;1(1):41-48.

33. Seiwert TY, Fayette J, Delcampo JM, et al. A randomized, openlabel phase II study of afatinib (BIBW 2992) versus cetuximab in patients with metastatic or recurrent squamous cell carcinoma of the head and neck (HNSCC) after failure of platinum-containing therapy with a cross-over period for progressing patients. Ann Oncol. 2010;21(suppl. 8):viii1-viii12. Proceedings of the $35^{\text {th }}$ ESMO Congress; Milan, Italy, October 8-12, 2010; abstract 1010PD.

34. Lin YL, Lin IC, Liou JC. Symptom patterns of patients with head and neck cancer in a palliative care unit. J Palliat Med. 2011;14(5):556-559.

35. Ang KK, Harris J, Wheeler R, et al. Human papillomavirus and survival of patients with oropharyngeal cancer. $N$ Engl J Med. 2010;363(1):24-35.

36. Rischin D, Young R, Fisher R, et al. Prognostic significance of $\mathrm{p} 16 \mathrm{INK} 4 \mathrm{~A}$ and human papillomavirus in patients with oropharyngeal cancer treated on TROG 02.02 phase III trial. J Clin Oncol. 2010;28(27):4142-4148.

37. Yung KC, Piccirillo JF. The incidence and impact of comorbidity diagnosed after the onset of head and neck cancer. Arch Otolaryngol Head Neck Surg. 2008;134(10):1045-1049.

38. Reid BC, Alberg AJ, Klassen AC, et al. Comorbidity and survival of elderly head and neck carcinoma patients. Cancer. 2001;92(8): 2109-2116.

39. Singh B, Bhaya M, Zimbler M, et al. Impact of comorbidity on outcome of young patients with head and neck squamous cell carcinoma. Head Neck. 1998;20(1):1-7.

40. Hathaway B, Johnson JT, Piccirillo JF, et al. Chemoradiation for metastatic SCCA: role of comorbidity. Laryngoscope. 2001;111(11 Pt 1):1893-1895.

41. Tanvetyanon T, Padhya T, McCaffrey J, et al. Prognostic factors for survival after salvage reirradiation of head and neck cancer. J Clin Oncol. 2009;27(12):1983-1991.

42. Rose BS, Jeong J-H, Nath SK, Lu SM, Mell LK. Population-based study of competing mortality in head and neck cancer. J Clin Oncol. 2011;29(26):3503-3509.

43. de Graeff A, de Leeuw JRJ, Ros WJ, Hordijk GJ, Blijham GH, Winnubst JA. Sociodemographic factors and quality of life as prognostic indicators in head and neck cancer. Eur J Cancer. 2001;37(3): 332-339.

44. Konski AA, Pajak TF, Movsas B, et al. Disadvantage of men living alone participating in Radiation Therapy Oncology Group head and neck trials. J Clin Oncol. 2006;24(25):4177-4183.

45. Terrell JE, Ronis DL, Fowler KE, et al. Clinical predictors of quality of life in patients with head and neck cancer. Arch Otolaryngol Head Neck Surg. 2004;130(4):401-408.

46. Pandey M, Devi N, Thomas BC, Kumar SV, Krishnan R, Ramdas K. Distress overlaps with anxiety and depression in patients with head and neck cancer. Psychooncology. 2007;16(6):582-586.

47. Brintzenhofe-Szoc KM, Levin TT, Li Y, Kissane DW, Zabora JR. Mixed anxiety/depression symptoms in a large cancer cohort: prevalence by cancer type. Psychosomatics. 2009;50(4):383-391.

48. Lazure KE, Lydiatt WM, Denman D, Burke WJ. Association between depression and survival or disease recurrence in patients with head and neck cancer enrolled in a depression prevention trial. Head Neck. 2009;31(7):888-892.

49. Howren MB, Christensen AJ, Karnell AH, Funk GF. Healthrelated quality of life in head and neck cancer survivors: impact of pretreatment depressive symptoms. Health Psychol. 2010; 29(1):65-71.

50. Wells N, Murphy BA, Dietrich M, Forastiere A, Li Y, Cella D. Quality of life and pain assessment for head and neck cancer patients treated on E1395: a comparison of two different Cisplatinbased chemotherapy regimens. Proc Am Soc Clin Oncol 21: abstract 907, p227a, 2002. 